\title{
Crystal structure of potassium bismuth hexathiodiphosphate, $\mathrm{KBiP}_{2} \mathrm{~S}_{6}$
}

\author{
V. Manriquez ${ }^{*, I}$, A. Galdámez , D. Ruiz León ${ }^{\mathrm{I}}$, M. T. Garland ${ }^{\mathrm{II}}$ and M. Jiménez ${ }^{\mathrm{II}}$ \\ I Universidad de Chile, Facultad de Ciencias, Departamento de Química, Casilla 653, Santiago, Chile \\ II Universidad de Chile, Facultad de Ciencias Físicas y Matemáticas, Departamento de Física, Casilla 487-3, Santiago, Chile
}

Received January 31, 2003, accepted and available on-line April 17, 2003; CSD-No. 409686

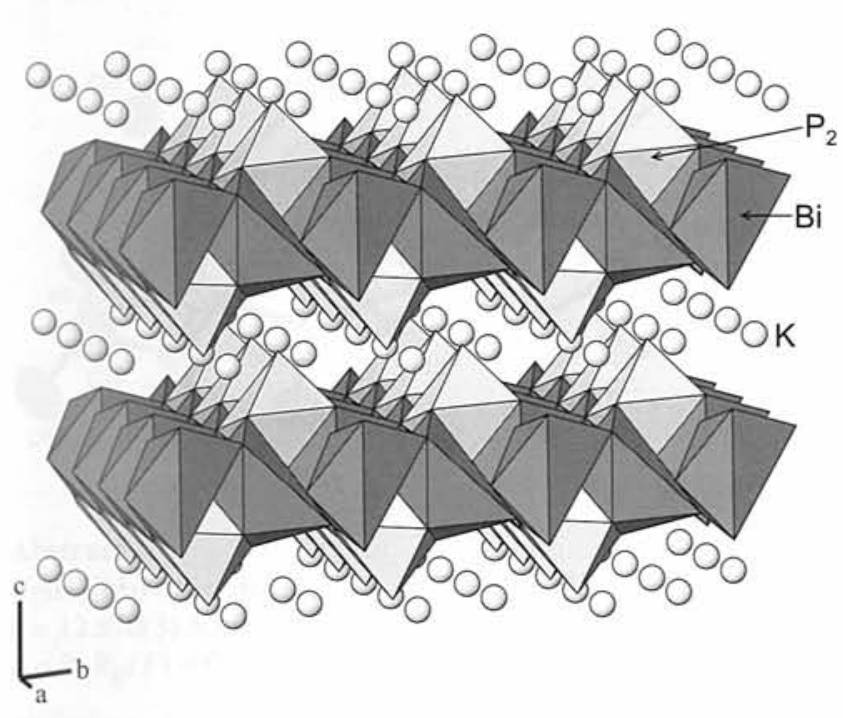

Abstract

BiKP $2 \mathrm{~S}_{6}$, monoclinic, $P 12_{1} 1$ (No. 4), $a=6.6200(6) \AA$, $b=7.4058(7) \AA, c=9.9002(9) \AA, \beta=92.108(1)^{\circ}, V=485.0 \AA^{3}$, $\mathrm{Z}=2, R_{\mathrm{gt}}(F)=0.027, w R_{\mathrm{ref}}\left(F^{2}\right)=0.060, T=293 \mathrm{~K}$.

\section{Source of material}

All manipulations were carried out under Ar atmosphere. For the synthesis of $\mathrm{KBiP}_{2} \mathrm{~S}_{6}$ stoichiometric amounts of the corresponding high purity (99.99\%) elements, supplied by Aldrich, were mixed an sealed in quartz tubes after evacuation. The mixture was them heated at $1123 \mathrm{~K}$ for two weeks. The reacted matter was slowly cooled $(0.4 \mathrm{~K} / \mathrm{min})$ to room temperature.

\section{Experimental details}

The absolute configuration was tested and the $w R\left(F^{2}\right)$ values for the two possible orientations were 0.0586 and 0.1907 . The first one represents the absolute structure of the crystal studied. The corresponding Flack parameter is $0.012(7)$.

\section{Discussion}

In the course of our work on chalcophosphate $[1,2]$ we have preparated the quaternary bismuth hexathiodiphosphate $\mathrm{KBiP}_{2} \mathrm{~S}_{6}$. The title compound has a complicated layered structure, which contains the ethane-like $\left[\mathrm{P}_{2} \mathrm{~S}_{6}\right]^{4}$ ligand. $\mathrm{KBiP}_{2} \mathrm{~S}_{6}$ is structurally related to $\mathrm{Na}_{0.16} \mathrm{Bi}_{1.28} \mathrm{P}_{2} \mathrm{~S}_{6}[3]$ and $\mathrm{KBiP}_{2} \mathrm{Se}_{6}$ [4]. The structure is characterized by layers of condensed $\mathrm{S}_{6}$ distorted octahedra alternately centered by $\mathrm{P}_{2}$ pairs and $\mathrm{Bi}$ atoms. The staircase layers of $\left[\mathrm{BiP}_{2} \mathrm{~S}_{6}\right]^{1-}$ groups in the $a-b$ plane are held together by a single layer of $\mathrm{K}^{+}$ions. The bond lengths in the hexathiodiphosphate $\left[\mathrm{P}_{2} \mathrm{~S}_{6}\right]^{4-}$ are $d(\mathrm{P}-\mathrm{P})=2.219(3) \AA$ and $d(\mathrm{P}-\mathrm{S})$ in the range from 1.962 (3) $\AA$ to 2.058 (3) $\AA$. The ethane-like $\left[\mathrm{P}_{2} \mathrm{~S}_{6}\right]^{4-}$ ligand chelates to $4 \mathrm{Bi}$ atoms, the bond lengths $d(\mathrm{Bi}-\mathrm{S})$ range from 2.706 (2) $\AA$ to $3.143(2) \AA$.

Table 1. Data collection and handling.

\section{Crystal:}

Wavelength:

$\mu$ :

Diffractometer, scan mode: $2 \theta_{\max }$ :

$N(h k l)_{\text {measured, }} N(h k l)_{\text {unique: }}$

Criterion for $I_{\mathrm{obs}}, N(h k l) \mathrm{gt}$ :

$N(\text { param })_{\text {refined: }}$

Programs: red dark prism, size $0.075 \times 0.075 \times 0.2 \mathrm{~mm}$ Mo $K_{\alpha}$ radiation $(0.71073 \AA)$ $201.48 \mathrm{~cm}^{-1}$

Siemens SMART CCD, $\varphi / \omega$ $56^{\circ}$

3908,2071

$I_{\text {obs }}>2 \sigma\left(I_{\mathrm{obs}}\right), 1901$

91

SHELXL-97 [5], ATOMS [6]

Table 2. Atomic coordinates and displacement parameters (in $\AA^{2}$ ).

\begin{tabular}{llllllllrrr}
\hline Atom & Site & $x$ & $y$ & $z$ & $U_{11}$ & $U_{22}$ & $U_{33}$ & \multicolumn{1}{c}{$U_{12}$} & $U_{13}$ & $U_{23}$ \\
\hline $\mathrm{Bi}$ & $2 a$ & $0.79399(4)$ & $0.77391(6)$ & $0.98012(3)$ & $0.0245(2)$ & $0.0188(1)$ & $0.0223(2)$ & \multicolumn{1}{l}{$-0.0012(2)$} & $0.0009(1)$ & $-0.0048(2)$ \\
$\mathrm{K}$ & $2 a$ & $0.7017(3)$ & $0.0236(3)$ & $0.5016(2)$ & $0.032(1)$ & $0.035(1)$ & $0.026(1)$ & \multicolumn{1}{l}{$-0.0025(9)$} & $-0.001(1)$ & $0.0003(9)$ \\
$\mathrm{P}(1)$ & $2 a$ & $0.6876(3)$ & $0.3148(2)$ & $0.8258(2)$ & $0.017(1)$ & $0.014(1)$ & $0.013(1)$ & $-0.0003(7)$ & $-0.0014(8)$ & $0.0005(7)$ \\
$\mathrm{P}(2)$ & $2 a$ & $0.7956(3)$ & $0.5409(3)$ & $0.7003(2)$ & $0.015(1)$ & $0.0139(9)$ & $0.014(1)$ & $-0.0008(8)$ & $-0.0017(8)$ & $0.0004(8)$ \\
$\mathrm{S}(1)$ & $2 a$ & $0.6585(3)$ & $0.4287(3)$ & $0.0136(2)$ & $0.026(1)$ & $0.020(1)$ & $0.013(1)$ & $-0.0044(9)$ & $0.0015(9)$ & $-0.0015(8)$ \\
$\mathrm{S}(2)$ & $2 a$ & $0.9127(3)$ & $0.1262(3)$ & $0.8259(2)$ & $0.019(1)$ & $0.0153(9)$ & $0.020(1)$ & $0.0016(8)$ & $-0.0028(9)$ & $-0.0003(9)$ \\
$\mathrm{S}(3)$ & $2 a$ & $0.4251(3)$ & $0.2268(2)$ & $0.7458(2)$ & $0.018(1)$ & $0.020(1)$ & $0.020(1)$ & $-0.0050(7)$ & $-0.0033(8)$ & $-0.0004(8)$ \\
$\mathrm{S}(4)$ & $2 a$ & $0.0521(3)$ & $0.6229(3)$ & $0.8071(2)$ & $0.015(1)$ & $0.023(1)$ & $0.019(1)$ & $-0.0013(8)$ & $-0.0010(8)$ & $-0.0024(9)$ \\
$\mathrm{S}(5)$ & $2 a$ & $0.5845(3)$ & $0.7341(2)$ & $0.7305(2)$ & $0.021(1)$ & $0.019(1)$ & $0.021(1)$ & $0.0043(8)$ & $-0.0048(8)$ & $-0.0008(8)$ \\
$\mathrm{S}(6)$ & $2 a$ & $0.8230(4)$ & $0.4592(3)$ & $0.5134(2)$ & $0.029(1)$ & $0.031(1)$ & $0.013(1)$ & $-0.004(1)$ & $0.0017(9)$ & $-0.0020(9)$ \\
\hline
\end{tabular}

\footnotetext{
* Correspondence author (e-mail: vmanriqu@uchile.cl)
} 
Acknowledgments. This work was supported by FONDECYT through operating grants No. 1020683, "Beca Apoyo Tesis 2002" and by Fundación Andes with the single crystal diffractometer.

\section{References}

1. Ruiz-León, D.; Manriquez, V.; Kasaneva, J.; Avila, R.E.: Insertion of trivalent cations in layered $\mathrm{MPS}_{3}(\mathrm{M}=\mathrm{Mn}, \mathrm{Cd})$ materials. Mater. Res. Bull. 37 (2002) 981-989.

2. Galdámez, A.; Manriquez, V.; Kasaneva, J.; Avila, R. E.: Synthesis, characterization and electrical properties of quaternary selenodiphosphates: $\mathrm{AMP}_{2} \mathrm{Se}_{6}$ with $\mathrm{A}=\mathrm{Cu}, \mathrm{Ag}$ and $\mathrm{M}=\mathrm{Bi}$, Sb. Mater. Res. Bull. (2003), accepted.

3. McCarthy, T.; Kanatzidis, M.: Synthesis in molten alkali metal polythiophosphate fluxes. The new quaternary bismuth and antimony thiophosphates $\mathrm{ABiP}_{2} \mathrm{~S}_{7}(\mathrm{~A}=\mathrm{K}, \mathrm{Rb}), \mathrm{A}_{3} \mathrm{M}\left(\mathrm{PS}_{4}\right)_{2}(\mathrm{~A}=\mathrm{K}, \mathrm{Rb}, \mathrm{Cs} ; \mathrm{M}=\mathrm{Sb}, \mathrm{Bi})$, $\mathrm{Cs}_{3} \mathrm{Bi}_{2}\left(\mathrm{PS}_{4}\right)$ and $\mathrm{Na}_{0.16} \mathrm{Bi}_{1.28} \mathrm{P}_{2} \mathrm{~S}_{6}$. J. Alloys Comps. 236 (1996) 70-85.
4. Breshears, J.; Kanatzidis, $\mathrm{M} .: \beta$ - $\mathrm{KBiP}_{2} \mathrm{Se}_{6}(\mathrm{M}=\mathrm{Sb}$, Bi): Kinetically accessible phases obtained from rapid crystallization of amorphous precursors. J. Am. Chem. Soc. 122 (2000) 7839-7840.

5. Sheldrick, G. M.: SHELXL-97. Program for crystal structure refinement. University of Göttingen, Germany 1997.

6. Dowty, E.: ATOMS (V 4.1). A Complete Program for displaying Atomic structures. By Shape Software, Kingsport, USA 1998. 